\title{
Kendal Care Home Project 2014-2016
}

\section{Alison Nicholson, Core Team Lead Nurse/Advanced Nurse Practitioner Kendal Integrated Care Community}

\section{BACKGROUND}

Kendal is a small market town in the South Lakes locality, with 3 GP practices covering the 37,000 population and outlying rural areas. At the beginning of 2014 there were 7 residential, nursing and dementia focussed care homes, with a total of 489 beds (of these 95 specific learning disability beds were not included in the pilot). In the latter stages of the pilot a new care home opened with an additional 38 residential beds, giving a new total of 527 beds.

The opening of a new purpose built 120 bedded home underpinned the need to explore proactive and joined up working between all 3 GP practices to support and manage the health of residents, many who move from different areas to be closer to family.

There was an overarching need to explore and reduce hospital admissions by $20 \%$. There is positive evidence (Quest for Quality BGS, 2011) that a structured, proactive approach to identifying and managing health needs in this group, with coordinated teams working together can lead to improved quality of care, reduced admissions, more appropriate prescribing and reduced prescribing costs. There is an approximated $50 \%$ turnover of these residents every year and many patients arrive at care homes with inadequate clinical and care information.

The project formally commenced on the 6th January 2014, initial staffing comprised a WTE (whole time equivalent) Nurse Practitioner (NP), and 0.5 WTE Pharmacist, shared by 2 pharmacists seconded from a partner organisation. The team explored many different ways of approaching and delivering the pilot, with a consensus from all partners that it would be valuable to spend a given length of time within each home. This fostered the need to conduct medication reviews, comprehensive geriatric assessments and advance care planning as many residents required support with choices as they approached the end of their lives.

\section{PHASE 1}

The beginning of 2014 saw a month's intensive support and intervention in a large nursing, residential and Elderly Mental Infirm (EMI) setting. This provided a positive experience and an excellent platform to gain an understanding of the care system and what quality care represents.

The following month reflected a mixed experience across 2 differing residential settings. This revealed healthier, more articulate residents with higher acute hospital admission rates and a distinct lack of case management. March replicated this with work in 2 different residential providers. Once again contrasting data was captured within health and well-being outcomes and differing levels of dependency and care evident.

Early data in this phase suggested that a total of 9 admissions were avoided, coupled with unquantifiable proactive interventions that may prevent future need for acute care. A vast number of end of life conversations were undertaken, which were recorded using the deciding rights document, this practice will promote person centred planning and the overall reduction in admissions and achievement of preferred place of care.

During the NP time in practice, GP visits were reduced by $80 \%$ when compared with the same period the previous year. Time was spent screening new residents and those discharged from hospital. Completion of the Comprehensive Geriatric Assessment tool was undertaken to provide a baseline health status and identify those who may be in the last year of life.

Medication reviews were completed by prescribing pharmacists, with reductions in artificial nutritional feeds, creams, analgesics and sedatives. Dementia drugs were explored, with trials of drugs and careful monitoring of residents undertaken. Overall almost $£ 11,000$ has been saved across 5 care homes, with the anticipation of further savings in future. In contrast, a number of mobile residents have been commenced on calcium and vitamin D supplements to protect osteoporotic bones and reduce fracture risk.

One residential home continued to have end of life discussions with their residents and families. They then requested support in completing plans, with a further 4 being completed.

A presentation to the South Lakes Clinical Commissioning Group (CCG) executive board detailing the pilot's early outcomes was delivered. From this, we were asked to outline some quick wins in terms of prescribing practice for care home residents. This included reduction in creams and nutritional supplements and in ensuring that there was a significant evidence based rationale for prescribing requests.

Overall phase one was hugely successful, with positive resident stories and the clear identification of a need for management for this vulnerable group.

\section{PHASE 2}

Phase 2 began in May 2014, with 6 weeks of intensive intervention within one of the town's biggest providers. Work commenced in a large mixed general nursing and dementia care home where it was clear that the home clearly catered for some of the most complex residents the project encountered, with many placed from other areas including Barrow, West Cumbria, Blackpool and Fylde coast and Carlisle.

During this phase it was evident that the use of an internet based pharmacy, coupled with two other pharmacy providers compromised the medicines safety of the residents. As a team, we reflected on this experience and concluded that we all required further support with the administrative element of prescribing, namely issuing prescriptions in a timely manner and ensuring faxed communication to care homes and pharmacies. A request was made to the CCG to realign project monies attached to an unfilled pharmacy technician role and convert it to Medicines Management (MM) support, this was agreed. The role was fulfilled by 2 ladies who had an existing MM role in GP practices and whom wanted to support the project with extra basic hours. The support was invaluable and has also provided the team with the ability to support care homes in ordering, hence reducing duplication and waste. 
This particular home was noted to have large stocks of artificial and prescribed feeds, some of the feeds had expired, some were assigned to deceased residents and some had been commenced without dietetic review and as such were not clinically indicated. An audit revealed $£ 585.23$ of unallocated supplements, with a further $£ 288.52$ with an expired date. The team have also been able to audit stocks of dressings, creams and catheter supplies with interesting results. From this snap shot we made a commitment to reduce waste stock and over ordering in all 7 homes, with this role being fundamental to the MM duties.

In addition to the MM role we also expanded our team to incorporate an Assistant Practitioner (AP) 6 hours each week. Initially we utilised her skill set to explore resident pathways around the myriad of services that are provided by many different partners, however following reflection and evaluation of this role, we decided to use her skills in the proactive management of chronic disease, to further reduce hospital admissions and optimise resident health. Whilst this caused some initial uncertainty within the project, as we were aware not to fill gaps and allow the project to creep and begin to offer a service, it was decided that this would be a proactive intervention and allow for nursing and care staff to develop skills in a timely manner.

The summer months saw the project team move to a large provider of residential/nursing EMI care home. The home had recently transfered the managerial role to the deputy. During our time in the home, a Care Quality Commission (CQC) inspection was conducted; they considered the project an 'example of good practice' and included this in their published report.

In the 6 week intervention in this home, the hospital admission rate was noted to be high, with 8 admissions despite ANP support. Two of the admissions were directly attributable to my assessment, with one resident having a stroke and another stepped up to Westmorland General Hospital (WGH) with delirium due to infection. Five were 999 admissions due to falls, peri-rectal (PR) bleed and chest pain and the remaining one was a GP facilitated acute admission. The use of Cumbria Health on Call (CHOC) services in this home was very high, although accurate figures were not supplied. This data represents the highest admission rate of all the care homes during the project intervention. Further analysis revealed that some residents on the residential unit in this home had a degree of unstable health and therefore sought acute admission and treatment. Some levels of advance planning discussions were conducted to document resident's wishes, in an attempt to reduce this in the future.

From the time spent in all care home settings, we were able to identify a distinct gap in education and training. This included basic training such as manual handling, infection prevention and techniques in managing conflict. In addition to this many of the nurses required the teaching of new skills in catheterisation, venepuncture and verification of expected death. The pilot team worked tirelessly in signposting managers towards free training and offered to shadow and mentor anyone who may need practical support.

In summary, phase 2 of the project provided an insightful and challenging opportunity to work alongside new providers. From a project perspective I consider that the health and well-being of the residents in these 2 homes was enhanced, and the quality of health care they received was switched from reactive to proactive. The project team during both phases 1 and 2 developed a working knowledge of the health of residents across all 7 homes, paying attention to the most vulnerable and those nearing the end of their lives. Gratitude and testimonials from residents and families is expressed on a daily basis for the enhanced level of support have been received, thus assisting in achieving a positive quality element of this work.

\section{PHASE 3}

Phase 3 of the project began on the 1st September 2014 and continued right through until the project ceased in June 2016. It represented an entirely different way of working with plans to spend one full day each week in larger homes and half days in smaller providers.

The beginning of this phase was very busy, as the phase one homes had many new residents requiring comprehensive geriatric assessment, advance care planning and medication optimisation. The team worked diligently to meet this need.

\section{PROJECT STRENGTHS}

\section{Partnership Working}

This project provided an excellent opportunity to build links with partners and agencies to enhance resident care. Relationships were established with the acute hospital consultant geriatrician, Parkinson's Disease specialist nurse, and hospital discharge lead and dementia matron. This work has provided several care home residents with a positive experience and rapid discharge from hospital, allowing them to return to their care home to die.

Joined up working with the community mental health team consultant psychiatrist and Care Home Education \& Support Service (CHESS) team practitioners was established. This facilitated the successful reduction in anti-psychotics in some individuals and early identification

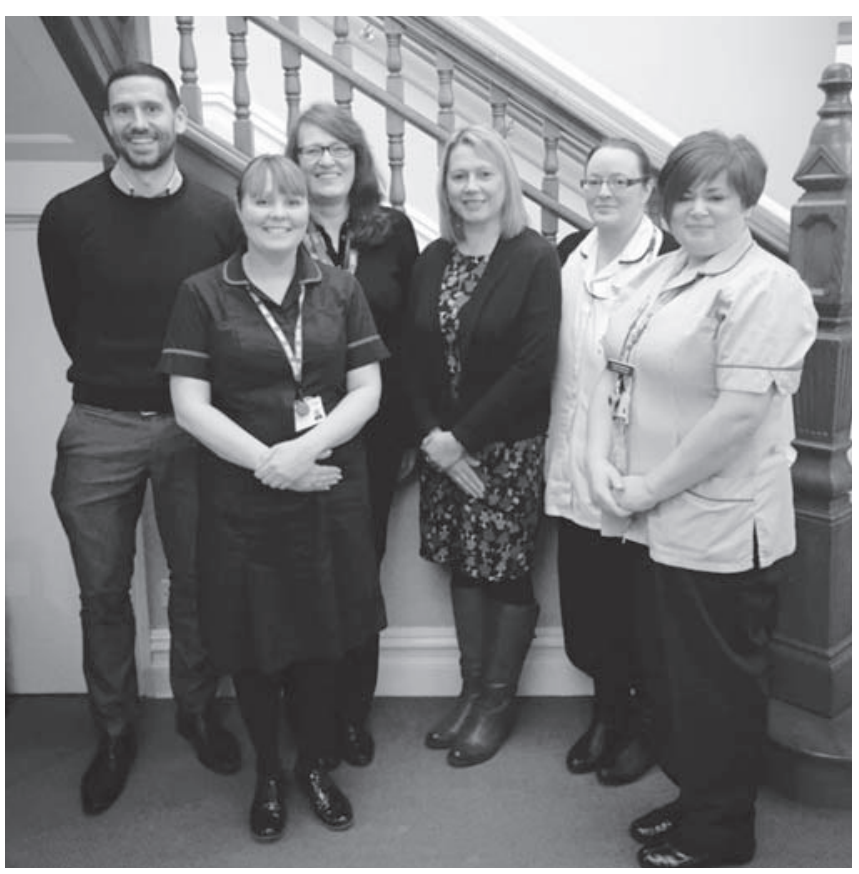

The Care Team 
of any deterioration in mental health. When time permitted we undertook monthly ward rounds together; thus enabling a proactive and holistic approach to resident assessment and support to the homes in managing complexity. The team were also able to support the teaching on the CHESS education programme, which provides a valuable opportunity to educate carers and nurses around physical illness, aspects of care and the signs to look for in dementia patients who are unwell.

I am extremely proud of the links and relationships we built during the past 2 and a half years, and firmly believe that during this time we were able enhance the quality of care for many of the residents and their family's experiences.

\section{Advance Care Planning Work}

Advance care planning and the use of 'Deciding Rights' documentation was undoubtedly an integral and successful part of this pilot. This work underpinned the whole project in terms of quality and dignity. Combined data from all phases reveals over 145 newly written 'Do not resuscitate' forms along with robust 'Emergency Health Care Plans' enabling clinicians to manage deterioration in an anticipatory manner. Many of these individuals have since died, with the formal data capture of hospital deaths vs care home deaths reflecting a positive shift to care home based deaths (see data below). This element of work continues to evolve in many aspects with our care home population, with GP colleagues now completing discussions and documents to reflect patient choices and wishes as their health deteriorates. This work will continue to be positively embedded and deliver positive resident outcomes, which will impact on the reduction of unplanned admissions.

\section{Death Rates (Care Home v Hospital) \\ 2013}

137 deaths, 118 in their own care home (86.2\%)

2014

100 deaths, 88 in their own care home (88\%)

\section{5}

121 deaths, 110 in their own care home (91\%)

\section{6}

129 deaths, 110 in their own care home (85.3\%, please note project ceased in June).

\section{Dietician Input}

Total $=30$ Residents on Feeds 18 Under Dietician $=60 \%$

Looking back in 2015, we identified a gap in the care of residents. A basic audit of prescribed dietary supplements (detailed above) revealed a staggering monthly spend of $£ 5,453$ from all 3 practices, across all 7 care homes. Of the residents receiving these prescriptions $40 \%$ had not been assessed by a dietician. It was clear that some of the supplements were added to prescriptions during hospital stays, and on other occasions care home staff rang GP practices to request supplements due to weight loss.

There is currently no commissioned dietician service within our locality and based on this we completed a proposal to the CCG to enable the care home project to commission one day each week of band 6 dietician support from the University Hospitals of Morecambe Bay NHS Trust (UMBHT) service. This role adopted a proactive nature, fostered by the pilot team, and included resident reviews, educational support and empowerment of staff with the aim to reduce the prescribing costs by $30 \%$ (approx. £18,000).

\section{IN SUMMARY}

This pilot spanning 2 and a half years undoubtedly proved to be successful in terms of resident health and wellbeing and positive end of life care. However in terms of true financial savings with regards to unplanned acute admissions and length of stay this is difficult to fully extrapolate.

Pharmaceutical reviews and saving amassed to an overall saving of $£ 93,794.17$. Within this area we have not represented the numbers of medications ceased due to palliative care and dysphagia, which again enhance the resident's quality of life.

The team were delighted to be finalists in 2 National Awards, the General Practice Awards 2105 and the BMJ Awards 2016. 